\title{
Situational Awareness Support to Enhance Teamwork in Collaborative Environments
}

\author{
Olga Kulyk \\ Human Media Interaction Group \\ University of Twente, \\ Drienerlolaan 5, 7522 NB \\ Enschede, the Netherlands \\ o.kulyk@ewi.utwente.nl
}

\author{
Gerrit van der Veer \\ Human-Computer Interaction \\ Open University, \\ Valkenburgerweg 177, 6419 AT \\ Heerlen, the Netherlands \\ gerrit@acm.org
}

\author{
Betsy van Dijk \\ Human Media Interaction Group \\ University of Twente, \\ Drienerlolaan 5, $7522 \mathrm{NB}$ \\ Enschede, the Netherlands \\ bvdijk@ewi.utwente.nl
}

\begin{abstract}
Motivation - Modern collaborative environments often provide an overwhelming amount of visual information on multiple displays. The multitude of personal and shared interaction devices leads to lack of awareness of team members on ongoing activities, and awareness of who is in control of shared artefacts. This research addresses the situational awareness (SA) support of multidisciplinary teams in co-located collaborative environments. This work aims at getting insights into design and evaluation of large displays systems that afford SA and effective teamwork.
\end{abstract}

Research approach - An exploratory (Wassink et al., 2008) as well as experimental approach is applied. The results of our exploratory studies, which included contextual observations, interviews and task analysis, have been translated into requirements for support of multidisciplinary teamwork in life sciences (Kulyk and Wassink, 2006). Currently we perform practical case studies in omics experimentation domain (Kulyk et al., 2007). In a first controlled study we assess shared SA of team members, providing new SA concepts on a shared large display.

Findings/Design - We developed several concepts for SA support on large shared displays. Memory Board is an interface that automatically stores and visualizes the activity history on a shared large display. This allows team members to retrieve annotations made on previous slides or visualizations. It also provides awareness of who is currently in control of any display, and who is manipulating and annotating the visualizations. Highlighting on Demand interface enables a team member to highlight or fade out any part of a display using any personal interaction device.

Take away message - Designing systems that support situational awareness is of great importance to ensure that a collaborative environment enables efficient and effective team coordination and decision making.

\section{Keywords}

Teamwork, situational awareness, collaborative working environment, shared displays, scientists.

\section{INTRODUCTION}

The diversity of multiple disciplines in teams positively impacts collaborative problem solving (Coughlan and Johnson, 2006). It is essential to analyse how such collaboration takes place in daily work practices. As the literature confirms, team collaboration can be supported by providing an appropriate environment and a certain context (Coughlan and Johnson, 2006). However, introducing a new environment and new technologies for example presenting multiple visualisations on a large display - may increase scientist's cognitive load and influence the way team members collaborate (Varakin et al., 2004). Awareness information in such shared workspace environments is required to coordinate team activities (Dourish and Bellotti, 1992).

The overwhelming amount of visual information on multiple displays, and the multitude of personal and shared interaction devices in new collaborative environments, lead to the lack of awareness of the ongoing activities, lack of understanding of shared visualisations and lack of awareness on who is in control of shared artefacts. The focus of our research is on the awareness support of co-located teams in collaborative working environments (Kulyk et al., 2007). Understanding who you are working with, what is being worked on, and how your actions affect others, is essential for effective team collaboration (Dourish and Bellotti, 1992). Such shared awareness helps teams to achieve goals that cannot be done by a single expert. Moreover, shared awareness also leads to informal social interactions and development of shared working cultures which are essential aspects of group cohesion.

\section{Situational awareness}

Situational awareness (SA) concerns "knowing what is (and has been) going on", being aware of what is happening around you in the environment and having a shared understanding of the information. Before giving a formal definition, we will first explain the importance of SA for team collaboration.

Situational awareness is expected to be an important determinant of team performance (Bolstad et al., 2005; Endsley, 1995). Especially in multidisciplinary settings situational awareness information is affected by abilities 
of individual members, their interaction with other team members, and the environment in which they collaborate (Bolstad et al, 2005). Various factors affect individual situational awareness formation: environmental (physical location, display arrangement and size etc.) and group aspects (communication, use of collaboration tools, team processes etc.). In order to assess SA during evaluation of collaborative interfaces or awareness displays, specific factors need to be identified relevant to a particular domain.

Situational awareness becomes even more critical in complex multi-display environments which change rapidly and provide a lot of detailed data. Recent studies (Brad et al., 2002; Rogers and Lindley, 2004) clearly point out that people are less aware of their visual surroundings than they think they are. Data overload, fatigue and other stressors can undermine the development and maintenance of the situational awareness (Bolstad et al., 2006). The phenomenon of change blindness shows that even if people have an accurate representation, they may still fail to notice changes (Martens, 2007, Varakin et al., 2004). Actively capturing attention at the location of the change by means of spatial cues improves the detection of the information and detection of changes. Therefore, it is of a great importance to design systems that support situational awareness and sharing of SA among team members to ensure efficient and effective team coordination and decision making.

Endsley's (1993, 1995) theory of situational awareness suggests that SA can be achieved by linking an objective state of the world to its mental analogue on three main levels: perception, comprehension and projection. Level 1 of $\mathrm{SA}$, is perception of relevant elements in the environment. It is an active process whereby individuals extract salient cues from the environment. Level 2 is comprehension of the meaning of these cues. It involves integration of information in working memory (Salas et al., 1995) to understand how it will impact the individual's goals and objectives. Level 3, projection, consists of extrapolating this information forward in time to determine how it will affect future states of the operating environment (Endsley, 1993). The third level of SA combines what the individual knows about the current situation with his or her mental model of similar events from previous experience, to be prepared for what might happen next.

In our research, we define $\mathrm{SA}$ as based on the three main aspects: (1) a person's previous knowledge and understanding of the situation, which contributes to identifying the source and nature of issues and problems; (2) detection and comprehension of the relevant perceptual cues and information from the environment, which supports comprehending multiple visualisations in their context; and (3) interpretation of these and reconfiguration of understanding and knowledge in a continuous process during the group collaboration effort. This allows awareness of changes in the environment, knowing what team members do and have done regarding current events in the environment, and keeping track of work progress.

Henceforward we refer to shared situational awareness as to the amount of communality of the individual SA of team members on the three aspects defined above. Our research investigates the following questions: What does situational awareness mean in team collaboration? How can we support situational awareness in collaborative working environments? How can shared displays support shared situational awareness in practice? How can we design and evaluate interactive systems and visualisations that afford situational awareness in order to stimulate existing and new forms of collaboration?

\section{AFFORDING SA IN SCIENTIFIC COLLABORATION}

In contrast to domains such as aircraft or plant operation control, emergency dispatch or crisis management (Mark, 2002; Sharma et al., 2003), scientific teams are not working in life-threatening situations and are not under constant strong time pressure. However, longterm scientific projects involve high costs and therefore errors are expensive to recover from. Shared visualisations on large displays have proven to be helpful to support group discussions because the support situational awareness (Rogers and Lindley, 2004). Other examples of teams using a large display to enhance awareness of their activities are software teams (Biehl et al., 2007).

\section{A First Application Domain for Empirical Research: Omics Experimentation}

Evolving technologies in molecular biology produce vast amounts of data. Scientists in this domain are confronted with the problem of applying methods from different disciplines when analyzing and interpreting their data, such as statistical, mathematical and machine learning techniques,. Moreover, integration of the results from heterogeneous information sources is a difficult part of their experiments' analysis. Current omics experimentation in molecular biology, for example in drug discovery and cancer research, is a complex, highly dynamic and multidisciplinary task that requires teamwork (Rauwerda et al., 2006; van der Vet et al., 2007). It is essential for life scientists to design the experiment precisely and accurately to insure the statistical validity of the data. Timely spotting of outliers and abnormal patterns in a huge amount of data is crucial for experimentation (see Figure 1). Recent studies showed that there is a strong need for visualising the omics datasets on a shared display for comparing and discussion among multidisciplinary scientists (Kulyk et al., 2007; Li et al., 2005).

Presenting visualisations on a shared display in a collaborative working environment can support group discussions (Rogers and Lindley, 2004). Looking at the statistical representations of the same data on a shared large display enables scientists to assess the quality of the entire omics experiment at a glance (Kulyk et al., 2007). The visualisations on the various parts of the display are implicitly related, in the sense that they refer 
to the same experiment, but currently it is not always evident what this precise relation is. To prevent team members from getting lost and to support situational awareness, the relations between various statistical representations have to be explicitly visualised. In order to afford detection of changes in visualisations and to avoid change blindness, it is important to draw team members' attention to current changes without distracting them from the discussion.

Multiple visualisations can be closely related, and therefore a change in a visualisation on one display will have to be related to visualisations on other displays. In our case, however, the situation is more complex. Scientists use discipline-related visualisations. For example, in microarray experimentation, spotting of outliers and abnormal patterns in the large data set can be done only by an expert in both statistics and in molecular biology, by analysing a combination of various statistical representations and microarray scans.

\section{Concepts for SA Support in Scientific Collaboration}

We are currently exploring various alternative solutions for SA support in collaborative environment for scientific teams (van der Vet et al., 2007). One example is a Highlighting on demand interface which enables the team member who is currently controlling the shared tiled display to draw attention of the team by highlighting a certain visualisation using a slider on a personal interaction device (for instance, TabletPC or a WiiMote controller).

Another concept is a Memory Board interface, which automatically stores and visualises the history of changes on a shared display, allowing team members to go back in time and retrieve annotations made on previous slides or visualisations. This board serves as a peripheral display that affords memorability and supports level 2 of situational awareness, comprehension.

We expect a supporting effect of visualisation of status information about who is in control of a display or another shared artefact on a personal interaction device. This would make every member of a team aware of who is making the changes and what changes are made. We intend to visualise the control interface on a shared touch display, as well as displaying it on a personal interaction device (e.g. Tablet PC). Such an interactive interface enforces sharing and thus supports coordination mechanisms and group awareness on who is currently manipulating and annotating the visualisations. It also partially resolves the potential control negotiation conflict about the annotation of visualisations and about manipulation of the shared display.

\section{ASSESSING SA SUPPORT IN COLLABORATIVE ENVIRONMENTS}

The complexity of communication processes in the colocated team environment requires the combination of several approaches to support situational awareness. This, in turn, requires a practical method to capture and analyse the dynamics of technology-mediated interactions in context. The nature of the interfaces as well as physical characteristics and affordances of the environment influence the way in which interactions occur (Fruchter and Cavallin, 2006). Therefore our approach for data analysis includes a combination of behaviour, interaction and environment analysis.

We will assess shared situational awareness of team members when we provide supportive visualizations on a shared large display. We aim at reducing disturbing factors that are considered distraction from the primary task. We intend to establish an indication of the relations between situational awareness, team satisfaction, group processes like decision making and the perceived task performance. In our case multiple data collection techniques are used: direct observations to assess user behaviour based on a validated coding scheme (Biehl et al., 2007), screen capturing, video recordings, questionnaires and post-interviews. Video recordings from several viewpoints combined with screen capturing of multiple displays, will enable us to analyse several simultaneously ongoing interactions. In addition to the observation coding scheme, postinterviews and questionnaires are carried out to obtain subjective judgements of the team members, e.g., on group satisfaction, awareness and distraction from primary tasks (Cadiz et al., 2002; Kulyk et al., 2006; Olaniran, 1996). Group satisfaction will be assessed by a combined validated post-questionnaire featuring the group process and decision making (Olaniran, 1996). We apply these questions to assess the perceived usefulness and impact of new Highlighting on Demand and Memory Board concepts on shared situational awareness of team members, on distraction from the primary task, and on team satisfaction with the group process and decision making process.

The three aspects of situational awareness described earlier, as well as recent related studies (Biehl et al., 2007; Blandford and Wong, 2004) will be used in defining relevant factors of SA in designing our questionnaire. We are adapting a computational model of shared situation awareness (Bolstad et al., 2005) to the context of our case studies. This model uses the Situation Awareness Global Assessment Technique (SAGAT) - an objective measure of situation awareness mainly based on work of Endsley (1995).

Our current observations and video analysis show that scientists tend to walk to the tiled display to inspect a specific detail of a visualisation, which indicates that they are treating the display different from a movie screen or a static projection. This points to the dynamic nature of interactions as reported in other studies (Tan et al., 2006). High resolution of the displays allows them to zoom in to a larger than life size image. Our observations indicate a high immersion, though possibly partially due to the novelty of the large displays.

Applying user study techniques and a multi-level method for data analysis will allow us to identify interaction patterns: natural ways in which team 
members interact with each other (behaviour patterns) and with the shared displays in the environment. Thus we may iteratively improve the design of SA support and construct a framework for the evaluation of how shared displays influence scientists' work and team collaboration.

\section{FUTURE WORK}

We will perform controlled comparative case studies on the impact of the Highlighting on Demand and Memory Board SA concepts. Our target group is small multidisciplinary team working on a joint project in life science domain. We will assess shared situational awareness of team members, providing supportive visualizations on a shared large display. We aim at reducing the distraction from the primary task, and establishing relations with team satisfaction, group process, decision making process, and with the perceived task performance.

In the second case study we aim at assessing the longterm influence of large shared display on team shared SA in other domain(s) and different collaborative environment(s). We will apply the adjusted measurements of shared SA from the first study. Crossculture and cross-organizational differences might show different effects compared to the first study.

\section{EXPECTED CONTRIBUTION}

This research aims at informing HCI theory and collaborative design practice on situational awareness support in shared workspaces, by presenting: (1) results of practical case studies on SA support demonstrating that: (a) SA has effect on group collaboration; and (b) SA can be manipulated; (2) an evaluation framework to assess situational awareness of multidisciplinary teams; and (3) implications for design of interactive systems and visualisations that afford shared situational awareness through awareness displays.

\section{ACKNOWLEDGMENTS}

We would like to thank the first author's supervisors Anton Nijholt, Paul van der Vet, collaborators Han Rauwerda, Wim de Leeuw, Tijs de Kler and other colleagues and friends for their helpful comments and ideas inspiring this research. This work is part of the BioRange program of the Netherlands Bioinformatics Center (NBIC), which is supported by a BSIK grant through the Netherlands Genomics Initiative (NGI).

\section{REFERENCES}

Biehl, J.T., Czerwinski, M., Smith, G. and Robertson, G.G. (2007) FASTDash: a visual dashboard for fostering awareness in software teams. In Proceedings of the SIGCHI conference on Human Factors in computing systems, San Jose, CA, USA. ACM Press. 1313-1322.

Blandford, A., Wong, B.L.W. (2004) Situation awareness in emergency medical dispatch. International Journal of Human-Computer Studies, 61:421-452.

Bolstad, C.A., Cuevas, H., Gonzalez, C., and Schneider, M. (2005) Modeling shared situation awareness. In Proceedings of the 14th Conference on Behavior Representation in Modeling and Simulation (BRIMS), Los Angles, CA, USA.
Bolstad, C.A., Costello, A.M., and Endsley, M.R. (2006). Bad situation awareness design: what went wrong and why. In Proceedings of the 16th World Congress of International Ergonomics Association, Maastricht, the Netherlands.

Brad, J., Armando, F., and Terry, W. (2002) The interactive workspaces project: Experiences with ubiquitous computing rooms. IEEE Pervasive Computing, 1(2):6774.

Cadiz, J. J., Venolia, G., Jancke, G., and Gupta, A. (2002) Designing and deploying an information awareness interface. In Proceedings of the ACM Conference on Computer Supported Cooperative Work (CSCW'02). ACM. NY. 314-323.

Coughlan, T. and Johnson, P. (2006) Interaction in creative tasks. In Proceedings of the SIGCHI conference on Human Factors in computing systems. ACM Press. 531540 .

Dourish, P. and Bellotti, V. (1992) Awareness and coordination in shared workspaces. In Proceedings of the ACM Conference on Computer-Supported Cooperative Work (CSCW '92), New York, NY, USA. ACM Press. 107-114.

Endsley, M.R. (1995) Measurements of situation awareness in dynamic systems. Human Factors, 37(1):65-84.

Endsley, M.R. (1993) A survey of situation awareness requirements in air-to-air combat fighters. International Journal of Aviation Psychology, 3(2):157-168.

Fruchter, R. and Cavallin, E. (2006) Developing methods to understand discourse and workspace in distributed computer-mediated interaction. AI and Society, 20(2):169-188

Kulyk, O., Wang, J., and Terken, J. (2006) Realtime feedback on nonverbal behaviour to enhance social dynamics in small group meetings. In Proceedings of the Joint Workshop on Multimodal Interaction and Related Machine Learning Algorithms (MLMI '06), vol. 3869, LNCS Series. Springer. 150-161.

Kulyk, O. and Wassink, I. (2006) Getting to know bioinformaticians: Results of an exploratory user study. In Adriaansen, T. and Zudilova-Seinstra, E., editors, In Proceedings of BCS HCI '06 Int, Workshop on Combining Visualisation and Interaction to Facilitate Scientific Exploration and Discovery, London, UK. 3037.

Kulyk, O., van Dijk, E.M.A.G., van der Vet, P.E., Nijholt, A. (2007) Do you know what I know? Situational Awareness and Scientific Teamwork in Collaborative Environments. In Proceedings Sixth International Workshop on Social Intelligence Design (SID), Trento, Italy, A. Nijholt, O. Stock and T. Nishida, editors, CTIT Workshop Proceedings Series, Vol. WP07-02, Enschede. 207-215.

Li, K., Hibbs, M., Wallace, G., and Troyanskaya, O. G. (2005) Dynamic scalable visualization for collaborative scientific applications. In Proceedings of the IPDPS '05 Workshop on Next Generation Software, Zurich. ACM Press. 162-169.

Mark, G. (2002) Extreme collaboration. Communications of the ACM, 45(6):689-93. 
Martens, M.H. (2007) The failure to act upon important information: where do things go wrong?Doctoral Thesis, Vrije Universiteit Amsterdam.

Olaniran, B.A. (1996) A Model of Group Satisfaction in Computer Mediated Communication and Face-to-Face Meetings, Behaviour and Information Technology, 15 (1):24-36

Rauwerda, H., Roos, M., Hertzberger, B. O., and Breit, T. M. (2006) The promise of a virtual lab in drug discovery. Drug Discovery Today, 11(5-6):228-36.

Rogers, Y. and Lindley, S. (2004) Collaborating around vertical and horizontal large interactive displays: which way is best? Interacting with Computers, 16(6):11331152 .

Salas, E., Prince, c., Baker, D.P., and Shrestha, L. (1995) Situation awareness in team performance: Implications for measurement and training. Human Factors, 37(1):123-136.

Sharma, R., Yeasin, M., Krahnstoever, N., Rauschert, I., Cai, G., Brewer, I., et al. (2003) Speech gesture driven multimodal interfaces for crisis management. In Proceedings of the IEEE, 91(9):1327-1353.

Van der Vet, P., Kulyk, O., Wassink, I., Fikkert, F., Rauwerda, H., van Dijk, E., van der Veer, G., Breit, T., and Nijholt, A. (2007) Smart environments for collaborative design, implementation, and interpretation of scientific experiments. In Huang, T., Nijholt, A., Pantic, M., and Pentland, A., editors, Proceedings of International Workshop on AI for Human Computing in conjunction with IJCAI' '07. 79-86.

Varakin, D. A., Levin, D. T., and Fidler, R. (2004) Unseen and unaware: Implications of recent research on failures of visual awareness for human-computer interface design. Human-Computer Interaction, 19(4):389-422.

Wassink, I., Kulyk, O., van Dijk, E.M.A.G., van der Veer, G., van der Vet, P.E. (2008) Applying a User-Centered Approach to Interactive Visualisation Design, In E.V. Zudilova-Seinstra, T. Adriaansen, and R. van Liere, editors, Trends in Interactive Visualisation, Advanced Information and Knowledge Processing series, Springer, $\mathrm{UK}$, in press. 